\title{
Assessing the value of matrix metalloproteinase 9 (MMP9) in improving the appropriateness of referrals for colorectal cancer
}

\author{
S Damery ${ }^{1}$, L Nichols ${ }^{2}$, R Holder ${ }^{2}$, S T Ward ${ }^{3}$, S Warmington ${ }^{1}$, S Wilson ${ }^{1}$, M J Wakelam ${ }^{4}$, J James ${ }^{5}$ \\ and $\mathrm{T}$ Ismail ${ }^{*, 3}$ \\ ${ }^{1}$ Primary Care Clinical Sciences, University of Birmingham, Edgbaston, West Midlands B15 2TT, UK; ${ }^{2}$ Primary Care Clinical \\ Research and Trials Unit, Primary Care Clinical Sciences, University of Birmingham, Edgbaston, West Midlands B15 2TT, UK; \\ ${ }^{3}$ University Hospitals Birmingham NHS Foundation Trust, Queen Elizabeth Hospital, Mindelsohn Way, Edgbaston, Birmingham \\ B15 2WB, UK; ${ }^{4}$ The Babraham Institute, Babraham Research Campus, Cambridge CB22 3AT, UK and ${ }^{5}$ Cancer Research UK Institute \\ for Cancer Studies, University of Birmingham, Edgbaston, Birmingham B15 2TT, UK
}

Background: A blood test may be an effective means of improving the appropriateness of referrals for symptomatic patients referred to specialist colorectal clinics. We evaluated the accuracy of a serum matrix metalloproteinase (MMP9) test in indicating colorectal cancer or its precursor conditions in a symptomatic population.

Methods: Patients aged over 18, referred urgently or routinely to secondary care following primary care presentation with colorectal symptoms completed a questionnaire and provided a blood sample for serum MMP9 estimation. Univariate analysis and logistic regression modelling investigated the association between presenting symptoms, MMP9 measurements and the diagnostic outcome of patient investigations, in order to derive the combination of factors which best predicted a high risk of malignancy.

Results: Data were analysed for 1002 patients. Forty-seven cases of neoplasia were identified. Age, male gender, absence of anal pain, diabetes, blood in stools, urgent referral, previous bowel polyps and previous bowel cancer were significantly associated with neoplasia. Matrix metalloproteinase 9 measurements were not found to be associated with significant colorectal pathology.

Conclusion: This study, despite robust sampling protocols, showed no clear association between MMP9 and colorectal neoplasia. Matrix metalloproteinase 9 therefore appears to have little value as a tool to aid referral decisions in the symptomatic population.

Colorectal cancer (CRC) is the third most common cancer in the United Kingdom, accounting for around $13 \%$ of new cancer cases each year (Cancer Research UK, 2010a). The annual total cost of diagnosing and treating CRC in England is estimated to be $\sim £ 1.1$ billion, with the cost of diagnosis forming the largest component of this expenditure (University of York Health Economics Consortium and University of Sheffield, 2007). In 2009, there were over 40000 new cases of CRC diagnosed in the United Kingdom, and over 16000 deaths (Cancer Research UK, 2010b). Survival is closely associated with stage at diagnosis; the current 5year relative survival rate for patients diagnosed with Dukes stage A is over $90 \%$, compared with $<7 \%$ for those diagnosed with latestage CRC (National Cancer Intelligence Unit, 2009). A national programme of biennial CRC screening to detect CRC in

\footnotetext{
*Correspondence: Dr T Ismail; E-mail: tariq.ismail@uhb.nhs.uk
}

Received 13 November 2012; revised 11 January 2013; accepted 16 January 2013; published online 7 February 2013 
asymptomatic individuals aged between 60 and 69 (extended in 2012 to include those aged up to 75) is now in operation (Atkin, 2006; Department of Health, 2007).

Despite the introduction of screening, the majority of CRCs continue to be diagnosed clinically because most cancers occur outside of the screening age range (Cancer Research UK, 2010a); around half of those invited to participate in screening currently decline (Logan et al, 2012) and screening cannot detect all cancers. Thus, many CRC patients are diagnosed following symptomatic presentation in primary care. Guidelines have been introduced to facilitate appropriate referral between primary and secondary care for patients with suspected cancer, with referral type (urgent or routine) determined by the symptoms reported and the clinical suspicion of malignancy (National Institute for Health and Clinical Excellence, 2005). All suspected cancer patients should be seen within 2 weeks of referral ('urgent referrals'); however, the application of guidelines may be problematic. The non-specific nature of many symptoms associated with CRC (Hamilton, 2009), and their variable interpretation by general practitioners mean that many patients referred as urgent do not meet referral guideline criteria; the majority of patients with bowel symptoms who meet criteria for urgent referral will not be found to have CRC, and some patients who are subsequently found to have cancer will have been referred routinely rather than urgently (Flashman et al, 2004).

There is some evidence that matrix metalloproteinases (MMPs), enzymes associated with tissue remodelling in both normal and pathological processes, could have potential application as tumour biomarkers (Collins et al, 2001). Overexpression of MMPs, and in particular MMP9, has been found in patients with colorectal adenomas (Parsons et al, 1998) and CRCs (Garbett et al, 1999; Heslin et al, 2001). Serum MMP9 measurements have been shown to be significantly higher in CRC patients compared with diseasefree controls, and higher in late-stage colorectal neoplasia compared with early stage disease (Baker and Leaper, 2003; Dragutinovic et al, 2011). If serum MMP9 concentration could be used to identify patients with significant colorectal pathology and malignancy, this would allow diagnostic investigations to be focused on the relevant patient population, and would reduce pressure on NHS resources within secondary care. It could also reduce the morbidity and mortality associated with subjecting a normal population to invasive tests such as colonoscopy and sigmoidoscopy.

We undertook a pilot study of 300 urgent referrals to a specialist colorectal clinic that showed that a predictive model incorporating serum MMP9 levels indicated colorectal neoplasia in $77.3 \%$ of cases (sensitivity 77.9\%, specificity 77.1\%) (Hurst et al, 2007). These results suggest that MMP9 may be an effective screening test, but the study was undertaken in a selected population with an unusually high prevalence of neoplasia and as such had limited generalisability. As the performance of a diagnostic test can vary according to a condition's severity and clinical presentation (Goehring et al, 2004), research into the association between MMP9 and colorectal neoplasia should be undertaken in a setting that provides a more representative spectrum of disease. This paper reports the findings of a large study that aimed to investigate whether a blood test that measured serum MMP9 levels could improve the appropriateness of referrals for symptomatic patients referred to a specialist colorectal clinic via either the urgent or routine referral pathways, and to determine the combination of factors (e.g., MMP9 and symptoms) that best predict whether a patient has CRC or its precursor conditions.

\section{MATERIALS AND METHODS}

Patient recruitment and data collection. The design of this study is reported in full elsewhere (Ryan et al, 2006). In brief, eligible patients were those aged 18 or over who were a new urgent or routine referral to the colorectal clinic at University Hospitals Birmingham NHS Foundation Trust within the study period. Eligible patients were identified via a search of Trust information systems, and those who met the inclusion criteria were sent an information leaflet and covering letter describing the research and a symptom questionnaire $\sim 2$ weeks before their scheduled clinic appointment. The questionnaire collected patient sociodemographic information; asked patients about bowel symptoms (derived from symptoms cited in the National Institute for Health and Clinical Excellence guidelines for referral of suspected CRC; NICE, 2005) and their duration; any injuries or other chronic illnesses experienced in the 3 months before questionnaire completion (as these can increase the levels of MMP9 in the blood), and collected data on personal or family history of CRC.

Invited patients who wished to take part in the study were asked to bring their completed questionnaire to their clinic appointment, and on arrival, those who indicated their desire to participate in the research were asked to see a research nurse prior to their appointment with the consultant. The research nurse answered any questions about the study and took consent, excluding any patients who were deemed unable to provide informed consent. Once a patient had consented to participation, the research nurse checked the questionnaire, providing non-directive assistance for completion of any unanswered questions and obtained a blood sample for MMP9 estimation. The results of each patient's clinic examination and any subsequent investigations were collected from hospital records using a standard proforma at least 2 months after the clinic appointment in an attempt to ensure that all relevant investigations had been completed and diagnoses documented. Patients without a definitive diagnosis within this 2-month period were followed up by study staff for as long as possible; all patients were tracked via the NHS Central Register (until April 2012) to maximise the ascertainment of malignancy in participating patients.

Storage and handling of blood samples. Blood samples were stored and handled in accordance with the study protocol. One 5$\mathrm{ml}$ blood sample was collected from each participating patient for MMP9 determination. Each sample was stored in a red-topped Vacuette tube (Greiner Bio-One Ltd., Gloucester, UK) with no additive, labelled, put into a plastic bag and kept on ice in an insulated polystyrene container until the end of the colorectal clinic. The container was then transported by hand to the laboratory at the Institute for Cancer Studies at the University of Birmingham and handed over to named personnel. Once received at the laboratory, each sample was immediately centrifuged and the serum fraction separated and stored at $-80^{\circ} \mathrm{C}$. Circulating MMP9 levels were determined on duplicate aliquots of each sample by ELISA assay kit (R\&D Systems, Abingdon, UK). No other haematology data were collected. The technician undertaking the ELISA assay was blinded to the symptoms and diagnosis of the patient to minimise bias, and all samples were analysed in the same laboratory to ensure that measurement and reporting were standardised.

Justification of sample size. The sample size calculation was based on the confidence interval and precision with which the sensitivity of MMP9 could be estimated. A sensitivity of $99 \%$ was obtained in study pilot work, and to estimate this sensitivity to within $2.5 \%$ precision (95\% confidence), blood samples from 60 people with colorectal neoplasia were required. Conservatively assuming a $6 \%$ prevalence of colorectal neoplasia (Thiis-Evensen et al, 1999; Lieberman et al, 2000), a sample of 1000 patients would allow a specificity of $63 \%$ to be estimated, with $4.0 \%$ precision $(95 \%$ confidence). Of these 1000 patients, we would expect 940 not to have colorectal neoplasia. 
Data analysis. Based on their 'final outcome' diagnosis, patients were classified into two pre-specified groups: a high-risk 'neoplasia' group consisting of patients diagnosed with adenocarcinoma of the colon or rectum, unspecified cancer of the colon/rectum, or highrisk polyps, and a low-risk 'non-neoplasia' group that consisted of patients diagnosed with low-risk polyps, cancer at sites other than colon/rectum, benign inflammatory disease of the colon/rectum, other benign disease of the colon/rectum, other benign conditions, or no abnormality.

All continuous variables (MMP9 measurements, age and body mass index (BMI) - derived from patient-reported height and weight) are summarised by medians and interquartile ranges (IQR) and univariate comparisons of these variables across the neoplasia/ non-neoplasia groups used the non-parametric Mann-Whitney $U$-test, as normality testing indicated that the data for these continuous variables were not normally distributed $(P \leqslant 0.01)$. Univariate odds ratios (OR) were calculated for each categorical sociodemographic, symptom-related and clinical variable to test the association between variable subgroups (e.g., presence or absence of a specific symptom) and colorectal neoplasia. In addition to analyses undertaken using MMP9 measurements as a continuous variable, an extra variable was created which categorised MMP9 by quintile.

Finally, binary logistic regression modelling was used to derive the combination of factors that best predicted a high risk of malignancy (e.g., invasive disease or high-risk polyps). All variables for which data had been collected were considered in the regression analyses. The model was derived using a backward elimination stepwise method whereby at each model iteration, variables with a statistical significance of $>0.2$ were removed, and the model rerun containing the remaining variables. Two regression models were generated: one in which MMP9 measurements were 'forced' to remain in the model, and one in which MMP9 measurements were removed from the model once the statistical significance of the MMP9 variable met the $>0.2$ criteria for variable removal. All statistical analyses were undertaken using Stata (version 11, StataCorp LP, College Station, TX, USA).

\section{RESULTS}

Response rates. Searches of appointment lists identified 2204 patients scheduled to attend the colorectal clinic who met the study inclusion criteria; all of whom were sent a study information leaflet and questionnaire prior to their appointment date. The flow of participants through the study is outlined in Figure 1. Of the 2204 patients invited to participate in the study, 185 (8.4\%) cancelled or did not attend their clinic appointment. Of the remaining 2019 eligible patients, 987 (44.8\%) either did not wish to see a research nurse about the study, or were unable to do so because their clinic appointment took place before a consent interview could be undertaken. Eight patients $(0.4 \%)$ were excluded from participation at the consent interview stage, leaving 1024 patients who gave consent to participate in the study. Of these, 20 patients did not give a blood sample, and a further two patients gave a blood sample which was unsuitable for analysis. The final number of study participants was 1002 (45.5\%).

A comparison of proportions test was undertaken to compare the characteristics (gender, age and referral type) of those consenting to participate in the study with those who did not participate. There was no significant difference between participants and non-participants on the basis of patient gender $\left(\chi^{2}=0.98 ; \mathrm{df}=1 ; P=0.322\right)$. Patients in both the youngest and oldest age groups (18-39 years and $70+$ years) participated in lower than expected numbers than those aged between 40 and 69 $\left(\chi^{2}=28.25 ; \mathrm{df}=3 ; P \leq 0.0001\right)$, and patients who had been referred

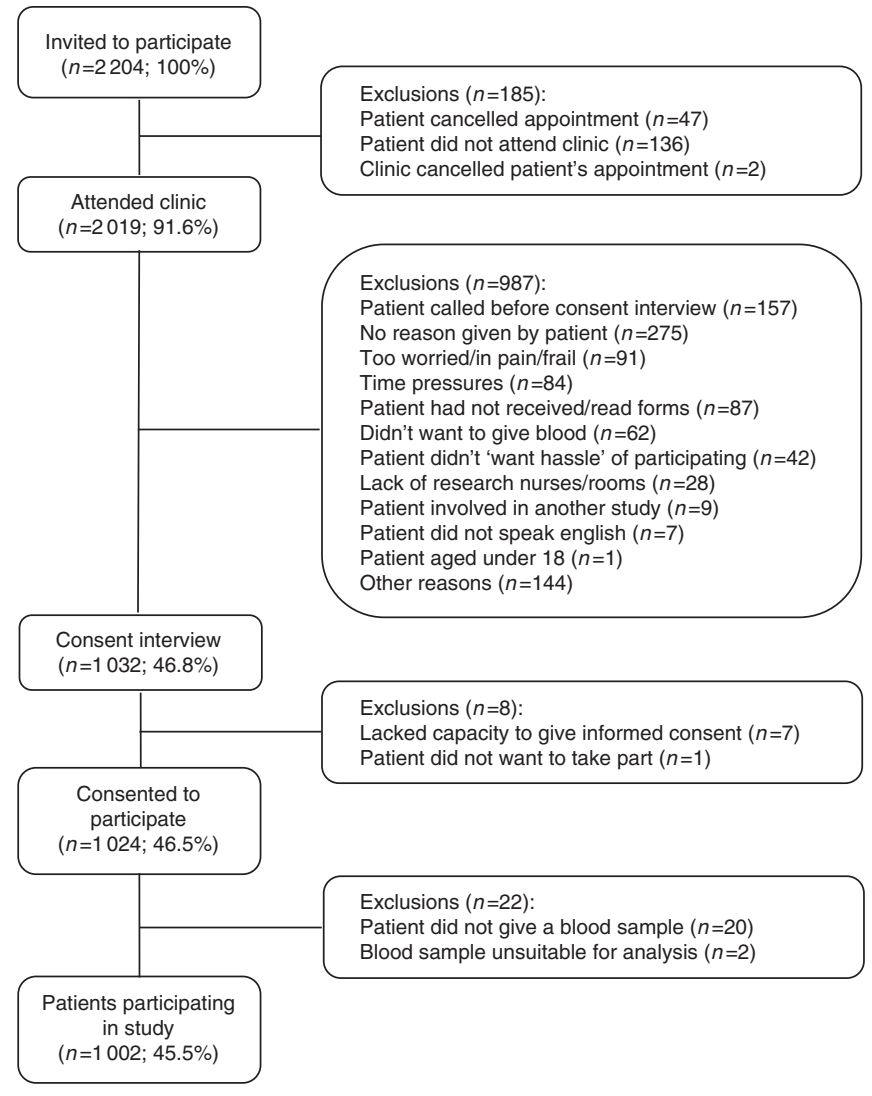

Figure 1. Patient recruitment flowchart.

as urgent were significantly more likely to participate in the study than those who had been referred routinely $\left(\chi^{2}=5.89 ; \mathrm{df}=1\right.$; $P=0.013)$.

Sample demographic and clinical characteristics. The median age of participants was 57 years (IQR: 43-68). In all, $48.7 \%$ of patients were males $(n=488)$, and the majority of participants were in the 'white' ethnic group $(n=891 ; 88.9 \%)$. Around a quarter of participants had been referred urgently $(n=249 ; 24.9 \%)$, (Table 1).

The most commonly reported colorectal symptoms were anal pain (54.5\%; median duration of symptoms: $14+$ weeks; IQR: 8-14 + weeks), tiredness (50.9\%; median duration: $14+$ weeks; IQR: 8-14 + weeks), change in bowel habit to looser stools (50.4\%; median duration: 12 weeks; IQR: $6-14+$ weeks), rectal bleeding (48.3\%; median duration: 12 weeks; IQR: $3-14+$ weeks), and the need to open bowels more frequently (44.9\%; median duration: 12 weeks; IQR: 6-14+ weeks). Symptoms reported by the fewest patients were weight loss $(n=142 ; 14.2 \%)$ and the need to open bowels less frequently ( $n=231 ; 23.1 \%)$, (Table 2$)$.

A number of patients reported co-morbid conditions within the 3 months prior to their participation in the study. In all, $32.0 \%$ $(n=321)$ had arthritis and 29.2\% (293) had high blood pressure. In all, 92 patients $(9.2 \%)$ reported a previous history of bowel polyps; 17 (1.7\%) had had a previous diagnosis of bowel cancer and 232 participants $(23.2 \%)$ reported a family history of bowel cancer.

Outcome of clinical investigations. Overall, 47 cases of neoplasia (30 colorectal adenocarcinomas, one other cancer of the colon/ rectum and 16 high-risk polyps) were identified as a result of investigations undertaken at or subsequent to, the clinic appointment, representing $4.7 \%$ of the study population. Other findings included 52 low-risk polyps (5.2\%), 22 cancers at sites other than 


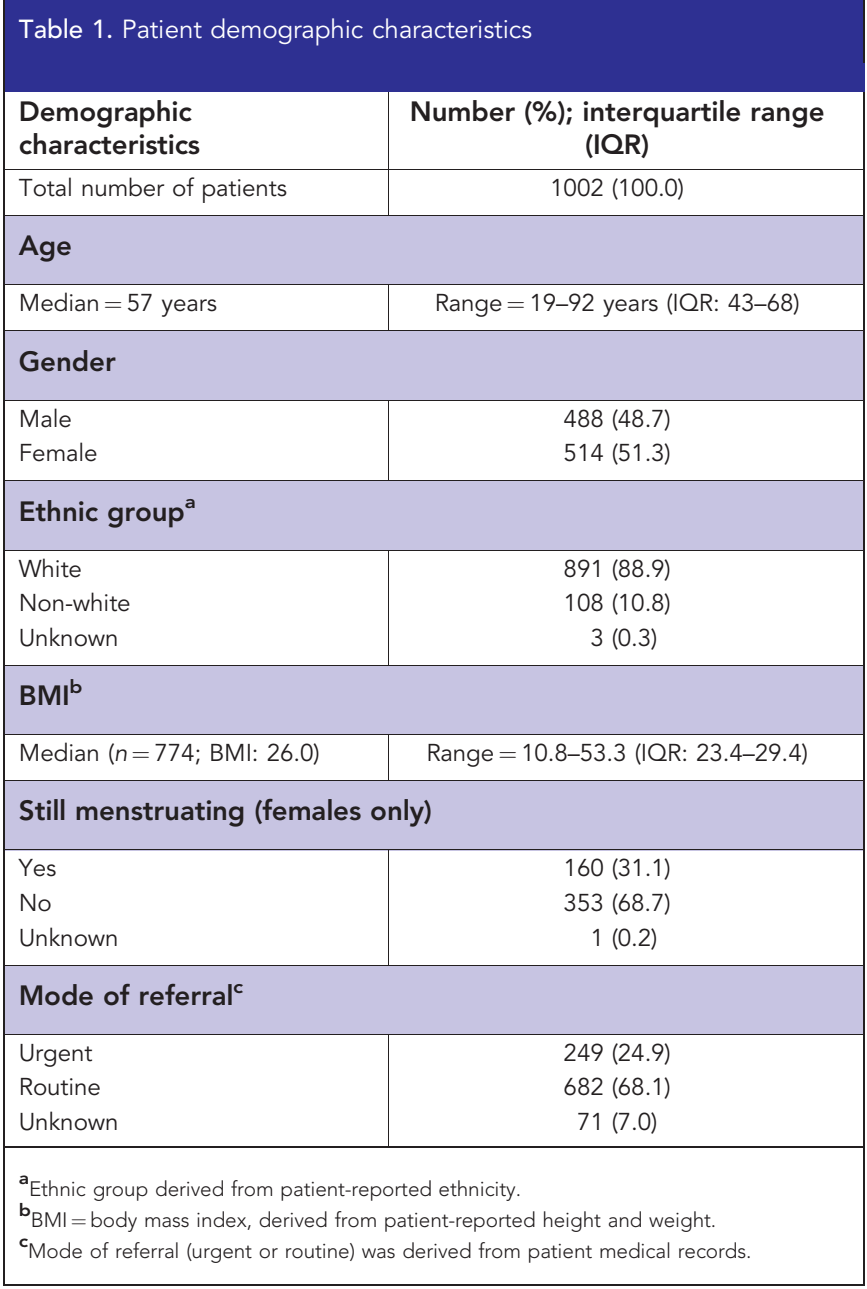

the colon/rectum (2.2\%) and 45 patients with inflammatory conditions of the colon/rectum (e.g., diverticulitis or inflammatory bowel disease (IBD); 4.5\%). No abnormality was found after investigation for 225 participants (22.5\%).

Serum MMP9 results. The median serum MMP9 concentration was $380.3 \mathrm{ng} \mathrm{ml}^{-1}$ (range: 99.9-1112.8; IQR: 236.4-578.8) in the neoplasia group, and $357.5 \mathrm{ng} \mathrm{ml}^{-1}$ (range: 52.5-1963.2; IQR: 221.3-560.1) in the non-neoplasia group, although this difference was not statistically significant (Mann-Whitney $U:$ 21637; $P=0.678$ ) (Table 3 ). In the univariate analyses undertaken to compare median MMP9 values between groups for each potential predictive factor (not tabulated), ethnicity was statistically significant (median MMP9 for the 'white' ethnic group $=369.2$ vs 306.9 for the 'non-white' group; $P \leqslant 0.001$ ), although it is likely that this finding is an artefact of the large difference in the number of individuals in each group (891 white $v s 108$ non-white patients). Median MMP9 concentrations were also significantly higher in patients reporting IBD than in those without it $(P=0.004)$. No other factors were statistically associated with median MMP9 concentrations.

Factors associated with the presence of colorectal neoplasia. Univariate analysis demonstrated that increasing age, male gender, the absence of anal pain, diabetes and blood in stools were significantly associated with the presence of neoplasia (Table 3 ). Other significant univariate predictors of neoplasia included being referred urgently (OR: 2.75 ; 95\% CI: 1.46-5.16), having had previous bowel polyps (OR: 4.26; 95\% CI: 2.16-8.40) and having had previous bowel cancer (OR: 12.66; 95\% CI: 4.48-35.71). Although we may expect that higher MMP9 concentrations would have a stronger association with neoplasia than lower concentrations, when categorised into quintiles, only MMP9 quintile two demonstrated a statistically significant association (OR: 2.92; $95 \%$ CI: $1.03-8.27$ ), with a $P$-value of 0.044 .

Entering all potential predictive sociodemographic and symptom-related factors into a backwards elimination stepwise logistic regression model, along with potential confounding factors such as recent injuries and co-morbidities (which could affect observed MMP9 concentrations) resulted in a final list of factors predictive of neoplasia in the study population (Table 4). The first model, in which MMP9 was forcibly retained, shows that gender, age, referral type, absence of tiredness, blood in stools, previous bowel polyps, previous bowel cancer and having an MMP9 measurement in quintile two were all statistically significant independent predictors of neoplasia. The pseudo $R^{2}$ (i.e., the proportion of variance between factors explained by the model) was 0.181 .

The second logistic regression model, in which MMP9 quintile was allowed to 'drop out' of the model when its statistical significance became $>0.2$, showed the same final list of independent predictive factors for neoplasia as the first. The $P$-values and OR for all factors retained in the model remained similar to their values in the first model, as did the pseudo $R^{2}$ for the model without MMP9 $\left(R^{2}=0.161\right)$.

\section{DISCUSSION}

This study investigated the association between serum MMP9 concentrations and colorectal neoplasia in a group of symptomatic patients referred from primary care to a secondary care colorectal clinic. In contrast to other work that has demonstrated a significant association between elevated serum MMP9 concentration and neoplasia in a group of urgently referred patients (Hurst et al, 2007), and a study in which serum MMP9 was found to be associated with neoplasia in a cohort of patients recruited from primary care on the basis of self-reported colorectal symptoms (Wilson et al, 2012), the present study did not find an association between serum MMP9 levels and neoplasia. This was despite the inclusion of MMP9 concentration as both a continuous and a categorical variable to enable the potential predictive value of MMP9 to be modelled more accurately.

The lack of an association observed here corroborates the findings of a recently published study which found that bloodbased biomarkers did not appear to be a viable alternative to existing screening and diagnostic tests used in detecting colorectal neoplasia (Tao et al, 2012). Similarly, Mroczko et al (2010) showed only a weak association between MMP9 and CRC in a study of 75 patients. Studies that have demonstrated an association between MMP9 and CRC have typically included healthy control groups (Biasi et al, 2012). Our study measured MMP9 levels in a group of patients in which MMP9 measurements would be useful as a screening test to detect significant colorectal pathology, rather than in patients where the diagnosis was already known.

Previous research has drawn attention to the need for robust sampling, handling and processing protocols when undertaking research using blood biomarkers (Jung, 2008). Strengths of this study include duplicate determination of serum MMP9 levels and dual data entry to ensure reliability of results. All sample processing was undertaken in the same laboratory and by the same technician. The use of serum MMP9 estimation has been criticised for showing increased MMP9 levels compared with plasma estimation (Jung, 2008), and the use of citrate plasma has been cited as the gold standard for estimating circulating MMP9 (Makowski and Ramsby, 2003). The use of clot activators in bloodsampling tubes for serum estimation has been shown to result in a 15-fold increase in serum MMP9 levels compared with that of 
Table 2. Patient clinical characteristics

\begin{tabular}{|l|c|c|c|c|}
\hline Characteristic $(n=1002)$ & Yes $(\%)$ & No (\%) & Missing (\%) & $\begin{array}{c}\text { Median duration } \\
\text { (IQR) }\end{array}$ \\
\hline
\end{tabular}

Patient-reported symptoms, duration and median duration in weeks ${ }^{a}$

\begin{tabular}{|c|c|c|c|c|}
\hline Abdominal pain & $433(43.2)$ & $563(56.2)$ & $6(0.6)$ & $12(4-14+)$ \\
\hline Weight loss & $142(14.2)$ & $846(84.4)$ & $14(1.4)$ & $14(6-14+)$ \\
\hline Tiredness & $510(50.9)$ & $483(48.2)$ & $9(0.9)$ & $14(8-14+)$ \\
\hline Blood in stools & $341(34.0)$ & $642(64.1)$ & 19 (1.9) & $8(2.5-14+)$ \\
\hline Rectal bleeding & $484(48.3)$ & $502(50.1)$ & $16(1.6)$ & $12(3-14+)$ \\
\hline Bowel habit - harder stools & $340(33.9)$ & $639(63.8)$ & $23(2.3)$ & $13(6-14+)$ \\
\hline Bowel habit - looser stools & $505(50.4)$ & $478(47.7)$ & 19 (1.9) & $12(6-14+)$ \\
\hline Open bowels less frequently & $231(23.1)$ & $745(74.4)$ & $26(2.6)$ & $12(5-14+)$ \\
\hline Open bowels more frequently & $450(44.9)$ & $539(53.8)$ & $13(1.3)$ & $12(6-14+)$ \\
\hline Anal pain & $546(54.5)$ & $447(44.6)$ & $9(0.9)$ & $14(8-14+)$ \\
\hline
\end{tabular}

Medical conditions (self-reported)

\begin{tabular}{|c|c|c|c|c|}
\hline Angina & $119(11.9)$ & $871(87.0)$ & $12(1.2)$ & - \\
\hline High blood pressure & $293(29.2)$ & $695(69.4)$ & $14(1.4)$ & - \\
\hline Diabetes & $73(7.3)$ & $914(91.2)$ & $15(1.5)$ & - \\
\hline Asthma & $165(16.5)$ & $826(82.4)$ & $11(1.1)$ & - \\
\hline Inflammatory bowel disease & $47(4.7)$ & $938(93.6)$ & $17(1.7)$ & - \\
\hline Arthritis & $321(32.0)$ & $659(65.8)$ & $22(2.2)$ & - \\
\hline Muscular problems & $144(14.4)$ & $843(84.1)$ & $15(1.5)$ & - \\
\hline Epilepsy & $20(2.0)$ & $968(96.6)$ & $14(1.4)$ & - \\
\hline Other medical conditions & $379(37.8)$ & $585(58.4)$ & $38(3.8)$ & - \\
\hline Injuries $<3$ months previously & $90(9.0)$ & $901(89.9)$ & $11(1.1)$ & - \\
\hline Previous bowel polyps & $92(9.2)$ & $888(88.6)$ & $22(2.2)$ & - \\
\hline Previous bowel cancer & $17(1.7)$ & $973(97.1)$ & $12(1.2)$ & - \\
\hline Family history of bowel cancer & $232(23.2)$ & $745(74.4)$ & $25(2.5)$ & - \\
\hline Haematology results & Number (\%) & Median & Range (IQR) & \\
\hline MMP9 (crude) $\mathrm{ng} \mathrm{ml}^{-1}$ & $1002(100.0)$ & 357.5 & $52.5-1963.2(221.3-560.1)$ & \\
\hline Outcome of clinical investigations & & Number (\%) & & \\
\hline $\begin{array}{l}\text { Adenocarcinoma of colon/rectum } \\
\text { Cancer not specified of colon/rectum } \\
\text { High-risk polyp }\end{array}$ & & $\begin{array}{r}30(3.0) \\
1(0.1) \\
16(1.6)\end{array}$ & & \\
\hline Total 'neoplasia' group & & $47(4.7)$ & & \\
\hline $\begin{array}{l}\text { Cancer at site other than colon/rectum } \\
\text { Low-risk polyp } \\
\text { Intraepithelial neoplasia at other site } \\
\text { Inflammatory benign condition of colon/rectum } \\
\text { Inflammatory benign condition at other site } \\
\text { Other benign condition of colon/rectum } \\
\text { Other benign condition at other site } \\
\text { No abnormality }\end{array}$ & & $\begin{aligned} & 22(2.2) \\
52 & (5.2) \\
2 & (0.2) \\
45 & (4.5) \\
12 & (1.2) \\
266 & (26.5) \\
331 & (33.0) \\
225 & (22.5)\end{aligned}$ & & \\
\hline Total 'non-neoplasia' group & & $955(95.3)$ & & \\
\hline
\end{tabular}

citrate plasma (Jung et al, 2005). However, the blood-sampling tubes used in this study did not contain any clot activators. The time between phlebotomy and centrifugation is also associated with elevated serum MMP9 levels in samples left at room temperature before centrifugation (Gerlach et al, 2007). In our study, samples were kept on ice until delivery to the processing laboratory, and our data showed no correlation between time to centrifugation and serum MMP9 levels (Pearson correlation, $r=-0.010 ; P=0.801)$.

The regression modelling undertaken in this study did not demonstrate that serum MMP9 levels have viable potential in predicting significant colorectal pathology. The model in which
MMP9 quintile was forcibly retained as a predictive variable did not show an improvement in the predictive value of the other variables retained in the final iteration of the model over and above that demonstrated by these variables in the model where MMP9 was removed. Despite this, it is reassuring to note that one of the strongest predictive factors for patients in the neoplasia group was the mode of referral, with those referred through the urgent pathway by their general practitioner being nearly three times more likely to be diagnosed with colorectal malignancy than those referred routinely.

An accurate biomarker for colorectal neoplasia would have potential as an alternative or secondary screening method to the 
Table 3. Factors considered predictive of neoplasia

\begin{tabular}{|c|c|c|c|c|c|c|}
\hline Variable & \multicolumn{2}{|c|}{ Number } & $\begin{array}{c}\text { Neoplasia } \\
\text { Median (IQR) }\end{array}$ & $\begin{array}{l}\text { Non-neoplasia } \\
\text { Median (IQR) }\end{array}$ & $\boldsymbol{U}$ & $\boldsymbol{P}$-value \\
\hline Age & \multicolumn{2}{|l|}{1002} & $66(54-76)$ & $57(43-68)$ & 15127 & 0.01 \\
\hline BMI & \multicolumn{2}{|l|}{774} & $26(24-29)$ & $26(23-29)$ & 21395 & 0.587 \\
\hline MMP9 & \multirow[t]{2}{*}{1002} & & $380(236-579)$ & $357(221-560)$ & 21637 & 0.678 \\
\hline Variable & & Category & Number & n (Row \%) & n (Row \%) & OR $(95 \% \mathrm{Cl}) ; \boldsymbol{P}$-value \\
\hline \multirow{2}{*}{\multicolumn{2}{|c|}{ Gender }} & Male & 488 & $30(6.1)$ & $458(93.9)$ & $1.91(1.04-3.52) ; 0.034$ \\
\hline & & Female & 514 & $17(3.3)$ & $497(96.7)$ & \\
\hline \multicolumn{2}{|l|}{ Ethnicity } & White & 891 & $43(4.8)$ & $848(95.2)$ & $1.31(0.46-3.70) ; 0.604$ \\
\hline & Non-white & 108 & $4(3.7)$ & $104(96.3)$ & \\
\hline \multicolumn{2}{|l|}{ Referral type } & Urgent & 249 & $20(8.0)$ & $229(92.0)$ & 2.75 (1.46-5.16); 0.001 \\
\hline & & Routine & 682 & $21(3.0)$ & $661(97.0)$ & \\
\hline Still menstruating & & Yes & 160 & $2(1.3)$ & $158(98.7)$ & $0.29(0.06-1.26) ; 0.098$ \\
\hline & & No & 353 & $15(4.2)$ & $338(95.8)$ & \\
\hline Abdominal pain & & Yes & 433 & $18(4.2)$ & $415(95.8)$ & $0.80(0.44-1.46) ; 0.464$ \\
\hline & & No & 563 & $29(5.2)$ & $534(94.8)$ & \\
\hline Weight loss & & Yes & 142 & $8(5.6)$ & $134(94.4)$ & $1.24(0.56-2.70) ; 0.596$ \\
\hline & & No & 846 & $39(4.6)$ & $807(95.4)$ & \\
\hline Tiredness & & Yes & 510 & $19(3.7)$ & $491(96.3)$ & $0.63(0.35-1.14) ; 0.127$ \\
\hline & & No & 483 & $28(5.8)$ & $455(94.2)$ & \\
\hline Blood in stools & & Yes & 341 & $24(7.0)$ & $317(93.0)$ & $2.04(1.13-3.66) ; 0.016$ \\
\hline & & No & 642 & $23(3.6)$ & $619(96.4)$ & \\
\hline Rectal bleeding & & Yes & 484 & $22(4.5)$ & $462(95.5)$ & $0.95(0.52-1.72) ; 0.861$ \\
\hline & & No & 502 & $24(4.8)$ & $478(95.2)$ & \\
\hline Bowel habit - harder stools & & Yes & 340 & $14(4.1)$ & $326(95.9)$ & $0.79(0.42-1.49) ; 0.467$ \\
\hline & & No & 639 & $33(5.2)$ & $606(94.8)$ & \\
\hline Bowel habit - looser stools & & Yes & 505 & $21(4.2)$ & $484(95.8)$ & $0.75(0.42-1.36) ; 0.348$ \\
\hline & & No & 478 & $26(5.4)$ & $452(94.6)$ & \\
\hline Open bowels less often & & Yes & 231 & $11(4.8)$ & $220(95.2)$ & $0.98(0.49-1.97) ; 0.965$ \\
\hline & & No & 745 & $36(4.8)$ & 709 (95.2) & \\
\hline Open bowels more often & & Yes & 450 & $19(4.2)$ & $431(95.8)$ & $0.80(0.44-1.46) ; 0.475$ \\
\hline & & No & 539 & $28(5.2)$ & $511(94.8)$ & \\
\hline Anal pain & & Yes & 546 & $18(3.3)$ & $528(96.7)$ & $0.49(0.27-0.90) ; 0.018$ \\
\hline & & No & 447 & $29(6.5)$ & $418(93.5)$ & \\
\hline Angina & & Yes & 119 & $7(5.9)$ & $112(94.1)$ & $1.30(0.57-2.97) ; 0.536$ \\
\hline & & No & 871 & $40(4.6)$ & 831 (95.4) & \\
\hline High blood pressure & & Yes & 293 & $19(6.5)$ & $274(93.5)$ & $1.65(0.91-3.01) ; 0.101$ \\
\hline & & No & 695 & $28(4.0)$ & $667(96.0)$ & \\
\hline Diabetes & & Yes & 73 & $8(11.0)$ & $65(89.0)$ & $2.76(1.24-6.13) ; 0.010$ \\
\hline & & No & 914 & $39(4.3)$ & $875(95.7)$ & \\
\hline Asthma & & Yes & 165 & $5(3.0)$ & $160(97.0)$ & $0.58(0.23-1.50) ; 0.262$ \\
\hline & & No & 826 & $42(5.1)$ & $784(94.9)$ & \\
\hline IBD & & Yes & 47 & $2(4.3)$ & $45(95.7)$ & $0.90(0.21-3.85) ; 0.890$ \\
\hline & & No & 938 & $44(4.7)$ & $894(95.3)$ & \\
\hline Arthritis & & Yes & 321 & $11(3.4)$ & $310(96.6)$ & $0.63(0.32-1.26) ; 0.194$ \\
\hline & & No & 659 & $35(5.3)$ & $624(94.7)$ & \\
\hline Muscular problems & & Yes & 144 & $5(3.5)$ & $139(96.5)$ & $0.69(0.27-1.76) ; 0.434$ \\
\hline & & No & 843 & $42(5.0)$ & $801(95.0)$ & \\
\hline
\end{tabular}




\begin{tabular}{|c|c|c|c|c|c|}
\hline Variable & Category & Number & $\begin{array}{l}\text { Neoplasia } \\
\text { n (row \%) }\end{array}$ & $\begin{array}{c}\text { Non-neoplasia } \\
\text { n (row \%) }\end{array}$ & OR (95\% Cl); $\boldsymbol{P}$-value \\
\hline \multirow[t]{2}{*}{ Epilepsy } & Yes & 20 & $0(0.0)$ & $20(100.0)$ & OR could not be calculated \\
\hline & No & 968 & $47(4.9)$ & $921(95.1)$ & \\
\hline \multirow[t]{2}{*}{ Other medical conditions } & Yes & 379 & $19(5.0)$ & $360(95.0)$ & $1.18(0.64-2.18) ; 0.591$ \\
\hline & No & 585 & $25(4.3)$ & $560(95.7)$ & \\
\hline \multirow[t]{2}{*}{ Injuries $<3$ months before } & Yes & 90 & $2(2.2)$ & $88(97.8)$ & $0.44(0.11-1.86) ; 0.44$ \\
\hline & No & 901 & $44(4.9)$ & $857(95.1)$ & \\
\hline \multirow[t]{2}{*}{ Previous bowel polyps } & Yes & 92 & $13(14.1)$ & 79 (85.9) & $4.26(2.16-8.40) ;<0.001$ \\
\hline & No & 888 & $33(3.7)$ & $855(96.3)$ & \\
\hline \multirow[t]{2}{*}{ Previous bowel cancer } & Yes & 17 & $6(35.3)$ & $11(64.7)$ & $12.66(4.48-35.71) ;<0.001$ \\
\hline & No & 973 & $40(4.1)$ & $933(95.9)$ & \\
\hline \multirow[t]{2}{*}{ Family history bowel cancer } & Yes & 232 & $9(3.9)$ & $223(96.1)$ & $0.77(0.37-1.63) ; 0.496$ \\
\hline & No & 745 & $37(5.0)$ & $708(95.0)$ & \\
\hline \multirow[t]{5}{*}{ MMP9 quintile } & 1 (Lower) & 200 & $5(2.5)$ & $195(97.5)$ & Reference \\
\hline & 2 & 201 & $14(7.0)$ & $187(93.0)$ & $2.92(1.03-8.27) ; 0.044$ \\
\hline & 3 & 200 & $7(3.5)$ & $193(96.5)$ & $1.42(0.44-4.53) ; 0.560$ \\
\hline & 4 & 201 & $12(6.0)$ & $189(94.0)$ & $2.48(0.86-7.16) ; 0.094$ \\
\hline & 5 (Higher) & 200 & $9(4.5)$ & $191(95.5)$ & $1.84(0.61-5.58) ; 0.283$ \\
\hline
\end{tabular}

Table 4. Binary logistic regression models for probability of neoplasia

\begin{tabular}{|c|c|c|c|}
\hline Variable & $\beta$ & $\boldsymbol{P}$-value & Odds ratio $(95 \% \mathrm{Cl})$ \\
\hline \multicolumn{4}{|c|}{ MMP9 included in model } \\
\hline Intercept & -6.579 & $<0.001$ & \\
\hline Gender & 0.731 & 0.046 & $2.08(1.01-4.26)$ \\
\hline Age & 0.030 & 0.012 & $1.03(1.01-1.05)$ \\
\hline Mode of referral & 1.000 & 0.005 & $2.70(1.35-5.42)$ \\
\hline Tiredness & -0.827 & 0.025 & $0.44(0.21-0.90)$ \\
\hline Blood in stools & 1.196 & 0.001 & $3.31(1.65-6.62)$ \\
\hline Anal pain & -0.501 & 0.161 & $0.61(0.30-1.22)$ \\
\hline Previous bowel polyps & 1.050 & 0.027 & $2.86(1.13-7.25)$ \\
\hline Previous bowel cancer & 2.009 & 0.007 & $7.45(1.72-32.36)$ \\
\hline \multicolumn{4}{|l|}{ MMP9 quintile } \\
\hline 2 & 1.234 & 0.046 & $3.44(1.02-11.57)$ \\
\hline 3 & 0.433 & 0.521 & $1.54(0.41-5.79)$ \\
\hline 4 & 1.216 & 0.057 & $3.37(0.96-11.81)$ \\
\hline 5 & 0.659 & 0.329 & $1.91(0.52-7.06)$ \\
\hline \multicolumn{4}{|l|}{ Pseudo $R^{2}=0.181$} \\
\hline \multicolumn{4}{|c|}{ MMP9 NOT included in model } \\
\hline Intercept & -5.764 & $<0.001$ & \\
\hline Gender & 0.722 & 0.046 & $2.06(1.01-4.19)$ \\
\hline Age & 0.028 & 0.016 & $1.03(1.01-1.05)$ \\
\hline Mode of referral & 1.042 & 0.003 & $2.84(1.43-5.63)$ \\
\hline Tiredness & -0.769 & 0.033 & $0.46(0.23-0.94)$ \\
\hline Blood in stools & 1.242 & $<0.001$ & $3.46(1.75-6.85)$ \\
\hline Anal pain & -0.423 & 0.231 & $0.66(0.33-1.31)$ \\
\hline Previous bowel polyps & 1.032 & 0.028 & $2.81(1.12-7.05)$ \\
\hline Previous bowel cancer & 1.786 & 0.014 & $5.97(1.43-24.91)$ \\
\hline Pseudo $R^{2}=0.161$ & & & \\
\hline
\end{tabular}

currently used screening modalities, which have relatively low patient acceptability, or as an aid to general practitioners in determining the most appropriate referral pathway for symptomatic patients. Although previous studies have found that serum MMP9 estimation may have some predictive value in indicating patients with colorectal neoplasia (Hurst et al, 2007; Wilson et al, 2012), these studies did not observe a large effect. The current study, which was undertaken on a large number of patients referred to secondary care for colorectal investigations, showed no clear association between MMP9 and colorectal neoplasia. Matrix metalloproteinase 9, therefore, appears to have little value as a tool to aid referral decisions in the symptomatic population.

\section{ACKNOWLEDGEMENTS}

We thank all of the men and women who participated in this study, and the staff at the colorectal clinic at University Hospitals Birmingham NHS Foundation Trust and staff (particularly research nurses) at the Wellcome Trust Clinical Research Facility at University Hospitals Birmingham NHS Foundation Trust. Ethical approval was obtained from North Birmingham Research Ethics Committee in October 2004 (Ref: 04/Q2704/29) and R\&D approval was given by University Hospitals Birmingham NHS Trust R\&D department in November 2004 (Ref: RRK 2656). Approval was also obtained from the Office for National Statistics for patient outcomes (cancer or death) to be 'flagged' by the NHS Central Register (now the Medical Research Information Service at the NHS Information Centre), Ref: MR1037.

\section{REFERENCES}

Atkin WS (2006) Impending or pending? The national bowel cancer screening programme. BMJ 332: 742. 
Baker EA, Leaper DJ (2003) The plasminogen activator and matrix metalloproteinase systems in colorectal cancer: relationship to tumour pathology. Eur J Cancer 39: 981-988.

Biasi F, Guina T, Maina M, Nano M, Falcone A, Aroasio E, Saracco GM, Papotti M, Leonarduzzi G, Poli G (2012) Progressive increase of matrix metalloproteinase- 9 and interleukin-8 serum levels during carcinogenic process in human colorectal tract. PLoS One 7(7): e41839.

Cancer Research UK (2010a) Bowel (colorectal) cancer - UK incidence statistics http://info.cancerresearchuk.org/cancerstats/types/bowel/ incidence/index.htm.

Cancer Research UK (2010b) Bowel (colorectal) cancer - UK mortality statistics http://info.cancerresearchuk.org/cancerstats/types/bowel/ mortality/index.htm.

Collins HM, Morris TM, Watson SA (2001) Spectrum of matrix metalloproteinase expression in primary and metastatic colon cancer: relationship to the tissue inhibitors of metalloproteinases and membrane type-1-matrix metalloproteinase. Br J Cancer 84(12): 1664-1670.

Department of Health (2007) NHS Cancer Reform Strategy. Department of Health: London.

Dragutinovic VV, Radonjic NV, Petronijevic ND, Tatic SB, Dimitrijevic IB, Radovanovic NS, Krivokapic ZV (2011) Matrix metalloproteinase-2 (MMP2) and -9 (MMP-9) in preoperative serum as independent prognostic markers in patients with colorectal cancer. Mol Cell Biochem 355: 173-178.

Flashman K, Leary DPO, Serapati A, Thompson MR (2004) The Department of Health's 'two week standard' for bowel cancer: is it working? Gut 53: 387-391.

Garbett EA, Reed MWR, Brown NJ (1999) Proteolysis in human breast and colorectal cancer. Br J Cancer 81: 287-293.

Gerlach RF, Demacq C, Jung K, Tanus-Santos JE (2007) Rapid separation of serum does not avoid artificially higher matrix metalloproteinase (MMP)9 levels in serum vs plasma. Clin Biochem 40: 119-123.

Goehring C, Perrier A, Morabia A (2004) Spectrum bias: a quantitative and graphical analysis of the variability of medical diagnostic test performance. Stat Med 23: 125-135.

Hamilton W (2009) Five misconceptions in cancer diagnosis. BJGP 59: 441-447.

Heslin MJ, Yan J, Johnson MR, Weiss H, Diasio RB, Urist MM (2001) Role of matrix metalloproteinases in colorectal carcinogenesis. Ann Surg 233: 786-792.

Hurst NG, Stocken DD, Wilson S, Keh C, Wakelam MJ, Ismail T (2007) Elevated serum matrix metalloproteinase 9 (MMP-9) concentration predicts the presence of colorectal neoplasia in symptomatic patients. $\mathrm{Br} J$ Cancer 97: 971-977.

Jung K (2008) Is serum matrix metalloproteinase 9 a useful biomarker in detection of colorectal cancer? Considering pre-analytical interference that may influence diagnostic accuracy. Br J Cancer 99: 553-554author reply 555.

Jung K, Meisser A, Bischof P (2005) Blood sampling as a critical preanalytical determinant to use circulating MMP and TIMP as surrogate markers for pathological processes. Int J Cancer 116: 1000-1001.
Lieberman DA, Weiss DG, Bond JH, Ahnen DJ, Garewal H, Chejfec G. for Veterans Affairs Cooperative Study Group (2000) Use of colonoscopy to screen asymptomatic adults for colorectal cancer. NEJM 343: $162-168$.

Logan RFA, Patnick J, Nickerson C, Coleman L, Rutter MD, von Wagner C. on behalf of the English Bowel Cancer Screening Evaluation Committee (2012) Outcomes of the bowel cancer screening programme (BCSP) in England after the first 1 million tests. Gut 61: 1439-1446.

Makowski GS, Ramsby ML (2003) Use of citrate to minimise neutrophil matrix metalloproteinase-9 in human plasma. Ann Biochem 22: 283-286.

Mroczko B, Groblewska M, Okulczyk B, Kedra B, Szmitkowski M (2010) The diagnostic value of matrix metalloproteinase 9 (MMP9) and tissue inhibitor of matrix metalloproteinases 1 (TIMP-1) determination in the sera of colorectal adenoma and cancer patients. Int J Colorectal Dis 25(10): $1177-1184$.

National Cancer Intelligence Unit (2009) Colorectal cancer survival by stage http://www.ncin.org.uk/publications/data_briefings/ colorectal_cancer_survival_by_stage.aspx.

National Institute for Health and Clinical Excellence (2005) Referral Guidelines for Suspected Cancer. NICE: London.

Parsons SL, Watson SA, Collins HM, Griffin NR, Clarke PA, Steele RJC (1998) Gelatinase (MMP-2 and -9) expression in gastrointestinal malignancy. $\mathrm{Br} J$ Cancer 78: 1495-1502.

Ryan AV, Wilson S, Wakelam MO, Warmington SA, Dunn JA, Hobbs FDR, Martin A, Ismail T (2006) A prospective study to assess the value of MMP-9 in improving the appropriateness of urgent referrals for colorectal cancer: study protocol. BMC Cancer 6: 251.

Tao S, Haug U, Kuhn K, Brenner H (2012) Comparison and combination of blood-based inflammatory markers with faecal occult blood tests for noninvasive colorectal cancer screening. Br J Cancer 106(8): 1424-1430.

Thiis-Evensen E, Hoff GS, Sauar J, Majak BM, Vatn MH (1999) Flexible sigmoidoscopy or colonoscopy as a screening modality for colorectal adenomas in older age groups? Findings in a cohort of the normal population aged 63-72 years. Gut 45: 834-839.

University of York Health Economics Consortium and University of Sheffield (2007) Bowel Cancer Services Costs and Benefits: Final Report to the Department of Health. University of York.

Wilson S, Damery S, Stocken DD, Dowswell G, Holder R, Ward ST, Redman V, Wakelam MJ, James J, Hobbs FDR, Ismail T (2012) Serum matrix metalloproteinase 9 and colorectal neoplasia: a community-based evaluation of a potential diagnostic test. Br J Cancer 106: 1431-1438.

This work is published under the standard license to publish agreement. After 12 months the work will become freely available and the license terms will switch to a Creative Commons AttributionNonCommercial-Share Alike 3.0 Unported License. 